\title{
Evaluating Complexity of Information System Architecture Using Fractals
}

\author{
Nary Subramanian \\ Department of Computer Science, The University of Texas at Tyler, \\ 3900 University Blvd., Tyler, TX 75799, USA \\ nsubramanian@uttyler.edu
}

\begin{abstract}
Information systems collect, process, store, and output information for the benefit of their stakeholders. They range in scale from a simple smart phone to the world wide web. An architecture of an information system represents a high-level view of a specific facet of the system. An important view of the information system is provided by its information architecture that allows stakeholders visualize transfer of information among system components. Evaluation of complexity of this information architecture gives stakeholders a means of selecting the best architecture from an information standpoint. In this paper we propose to use fractal dimension as a means of evaluating complexity of information architecture of systems. Fractals are recurring patterns at multiple scales and fractals can be evaluated by means of their dimensions. In this paper, we model the information architecture as a fractal, compute its dimension, and determine its complexity. We develop the theory for fractal complexity evaluation, propose algorithms for practical application, and apply them to evaluate complexity of architectures.
\end{abstract}

Keywords: Information, Architecture, Evaluation, Complexity, Fractal.

\section{Introduction}

An information system is an arrangement of people, data, processes, and information technology that interact to collect, process, store, and provide as output the information needed to support an organization [1]. There are several different types of information systems such as transaction processing system or management information system, and they range in application scale from a simple app on a smart phone, through desktop applications and enterprise applications, to the world wide web itself. An important aspect of an information system is that each of them possesses an architecture that defines the high level view of an aspect of the system [2]. Therefore, there exists a system architecture, information architecture, hardware architecture, and infrastructure architecture for an information system. System architecture defines the high-level interactions between hardware, software, people, and data within an information system; information architecture defines the high-level view of information sources and sinks within a system - information includes both data and control flows in the system; hardware architecture defines the interactions 
between hardware components in the system; while infrastructure architecture defines the interaction between software and the hardware components on which software execute. In this paper we investigate complexity analysis of information architecture for information systems.

The information architecture of an information system consists of components, connections, patterns, and styles [3]. Components are the sources and sinks of information including elements such as data readers, cameras, human data entry sources, and displays; connections are data and control flows; styles are the layout of components and connections such as layered, object-oriented, or pipe-and-filter; while patterns are the repeating motif in the architecture such as IPO (input-process-output) or MVC (model-view-control) pattern. There is a class of patterns for information architectures that make those architectures distinct from others - we call this the fractal pattern. Fractals are mathematical objects that have a very specific property of hierarchy wherein higher elements in the hierarchy subsume the pattern of the lower ones. For example, in Fig. 1a, one can see several levels of hub-and-spoke pattern for a games information system used when conducting modern large-scale sports events such as Olympics $[4,5]$. In this figure we can see several basic systems used for gathering athlete information in the field, including the False Start Control System, Automatic Photo Finish System, Transponder Control System, and Display Control System. These systems are connected to the respective physical devices such as the starter's gun, timers, video recording equipments, and display boards. These systems are each at level 1 in the hierarchy (indicated by $n=1$ in the figure) and they are connected to Competition Result System- which will be at level 2 in the hierarchy (indicated by $n=2$ in the figure). Similarly, at level 3, Competition Result System, Commentator Information System, Security System will all be connected to the Games Information System. In Fig. 1b, we see the repeater pattern (or transporter pattern) at several hierarchies for the human nervous system [6]: at level 1 we have axons carrying information between neurons, at level 2 we have nerves carrying information between sets of neurons and the spinal column, and at level 3 we have spinal column carrying information to the brain. It will be interesting to characterize the complexity of such information architectures using fractal complexity metrics.

Complexity is an important non-functional requirement for software architectures since the measure of complexity gives a good indication of the difficulty of maintaining the software system. Typically the more complex the software architecture the more difficult it is to maintain the software and since maintenance is the most expensive part of software operation, it will be of interest to software practitioners to have a measure of the complexity at the architecture level itself. Very few methods exist in literature for evaluating software architecture complexity. McCabe's cyclomatic complexity [7] is one of the oldest known methods for evaluating complexity when the detailed structure of software is known - however, since structure is typically not clear until detailed design, this method is not easily applied at the software architectural level. Extent of design pattern coverage in an architecture has been used to specify the complexity of architecture [8] using an Interactive Architecture Pattern Recognition System - however, patterns considered do not include the fractal types that is the subject of this paper. It has been proposed that architecture dependency graphs may be used for evaluating complexity [9] however, it is not always apparent whether all dependencies have been covered in this 
method. Likewise a function point method has been specified in [10] - but relationship between function points and software architectures is not always straightforward. In this paper we use the fractal dimension as a measure of complexity of self-similar information architectures.

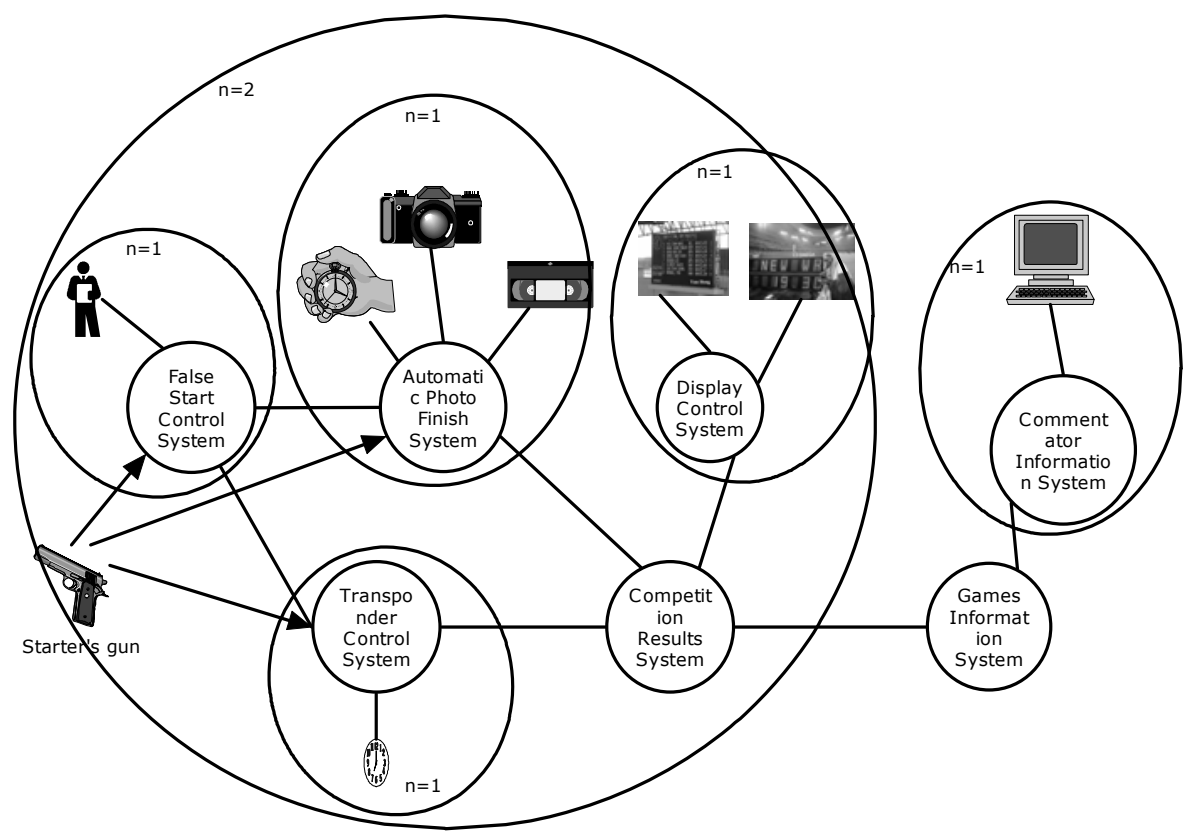

a. Two Fractal levels of Games Information System using Hub-and-Spoke Pattern

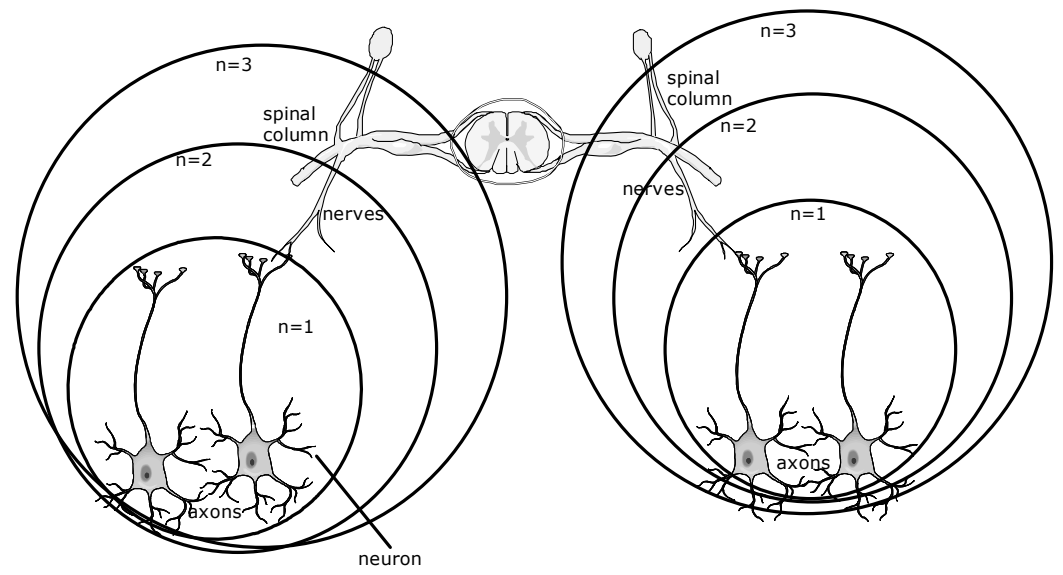

b. Three Fractal levels of Human Nervous System using Repeater (or Transporter) Pattern

Fig. 1. Fractal Nature of Certain Information Architectures 
This paper is organized as follows: Section 2 discusses evaluation of information architecture complexity with fractals, Section 3 presents algorithms for complexity evaluation and computes the complexity of two practical systems, Section 4 discusses issues with complexity evaluation using fractals, and Section 5 summarizes the approach and provides directions for future research.

\section{Fractal Math for Complexity Evaluation}

Fractals seem to follow an emergent nature. As investigated by Mandelbrot $[11,12]$, halving the unit of measurement for length does not double the size of the item measured, in fact it increases more than double the original measurement. This property of fractal fits in well with the measurement of complexity since being a nonfunctional requirement makes complexity an emergent property as well [16]. The equation for measuring length of a coastline [13] using fractals is given in eq. 0 ,

$$
P=k A^{D / 2} \text {. }
$$

where $P$ is the perimeter, $A$ is the area, $k$ is the constant of proportionality, and $D$ is the fractal dimension. As discussed in [14], $D$ is a good indicator of how complex the fractal is. For the purpose of evaluating complexity of information architectures we modify eq. 0 to the one in eq. 1 ,

$$
I=k N^{C / 2} .
$$

where $I$ is information, $N$ is the number of components in the architecture, $k$ is the constant of proportionality, and $C$ is the complexity of the architecture.

Taking, natural logarithm on both sides, the above equation may be rewritten as

$$
\ln I=\ln k+\frac{C}{2} \ln N .
$$

In eq. 2, the complexity $C$ is twice the slope of the line in the $\ln I$ and $\ln N$ plane. Therefore, if we plot a linear regression line in a plot on this plane, the slope of this line gives half the value of the complexity. Thus, we can define complexity of information architectures $C$ as

$$
C=2 \times \text { slope of regression line in logarithmic I and } N \text { plane. }
$$

Based on above definitions, we develop the following theorems.

Theorem 1. Complexity of Information Architectures $(C)$ will satisfy the relation $0 \leq C \leq \infty$.

Discussion: By eq. 3, $C$ is twice the slope of a regression line. Slope of a line follows the relation $0 \leq$ slope $\leq \infty$ and, therefore, $0 \leq C \leq \infty$. This is different from the case in [15] wherein fractal dimension is constrained between 1 and 2 for the topological domain.

Theorem 2. Complexity of Information Architectures $(C)$ with low throughput components will be closer to 0. Complexity of Information Architectures $(C)$ with high throughput components will be closer to $\infty$. 
Discussion: In eq. 2, when $I$ is small for large values of $N$, the slope of the line will be low making $C$ closer to 0 . Likewise, in eq. 2 , when $I$ is large for small values of $N$, the slope of line will be high making $C$ closer to $\infty$. This can be visualized better in Fig. 2 where the linear regression lines for both low throughput and high throughput environments are given.

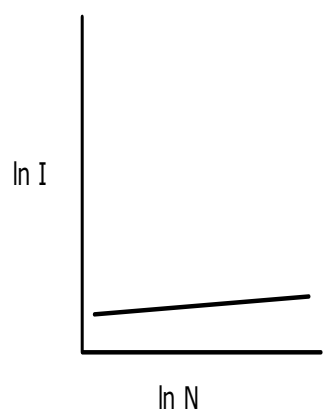

\section{A. Linear Regression in Low Throughput Environment}

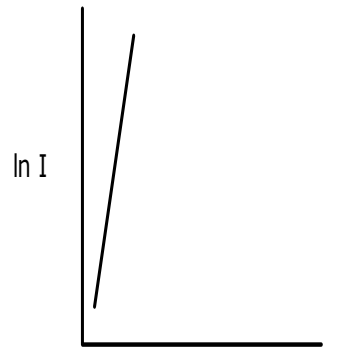

$\ln \mathrm{N}$

\section{B. Linear Regression in High Throughput Environment}

Fig. 2. Figures for Visualizing Theorem 2

Theorem 3. The constant of proportionality $k$ in eq. 2 equals the throughput in the trivial case, namely, when the architecture has only one component.

Discussion: In eq. 2 , when $N=1$, the resulting equation is

$$
\ln I=\ln k \Rightarrow k=I \text {. }
$$

Therefore, $k$ defines the initial condition of the system when only one component has been included in the system.

Theorem 4. Complexity of Information Architectures $(C)$ may be computed also by the following equation:

$$
C=2 \times \frac{\ln I^{\prime}}{\ln N^{\prime}}
$$

where $I^{\prime}$ is the maximum expected information throughput of the architecture corresponding to $N^{\prime}$, the maximum expected number of components in the architecture.

Discussion: Let's assume that components are added to the information architecture in the ascending order of their information throughput, where $I$ is the information throughput of the first component, and $I^{\prime}$ the information throughput when $N^{\prime}$ components have been added. Then, by eq. 2 and Theorem 3 , we have

$$
\ln I^{\prime}=\ln I+\frac{C}{2} \times \ln N^{\prime}
$$


This is same as,

$$
\ln \frac{I^{\prime}}{I}=\frac{C}{2} \times \ln N^{\prime} .
$$

When $I^{\prime} \gg I$, since items have been added in ascending order, eq. 7 can be written as,

$$
C=2 \times \frac{\ln I^{\prime}}{\ln N^{\prime}}
$$

Theorem 4 will allow us to develop the algorithm for evaluating complexity given in the next section.

\section{Evaluating Information Architecture Complexity Using Fractals}

There are two possible ways to evaluate complexity - where the information distribution patterns are known we can use eq. 3 to compute complexity and where the architectural elements' throughput are known we can use eq. 8 to compute complexity.

Algorithm 1: complexity evaluation when information distribution pattern is known:

1. Plot the data rates for the different devices on the logarithmic $I$ vs. logarithmic $N$ scale

2. Compute the linear regression that best fits the points plotted.

3. Complexity is, by eq. 3 , twice the slope of this regression line.

Algorithm 2: complexity evaluation when throughput of individual elements of the architecture are known:

1. Include all data sources and sinks in the information architecture.

2. Include all control actuators in the information architecture.

3. For each data source, data sink, and control actuator estimate the approximate data rates.

4. Compute the total number of data sources, data sinks, and control actuators as $N$.

5. Compute the total data rate as $I^{\prime}$.

6. Calculate the complexity of information architecture by eq. 8 .

\subsection{Evaluation of Complexity of Computer Lab Network}

The fractal pattern is also found in the computer network in a University. The data collected in the research lab at the Department of Computer Science at the University of Texas at Tyler at about 10 am on a weekday morning is shown in Table 1.

We apply Algorithm 1 above and obtain the graph of Fig. 3. The slope of the linear regression $(\ln I=3.4379+0.2882 \ln N)$ is 0.2882 , which gives the complexity of the information architecture to be 0.5764 (since, by eq. 3 , complexity is twice the slope). 
Table 1. Information Throughput in a Computer Lab Network

\begin{tabular}{|c|c|}
\hline No. of Nodes & Data in Kbps \\
\hline 1 & 50 \\
\hline 3 & 50 \\
\hline 4 & 120 \\
\hline 5 & 50 \\
\hline 6 & 75 \\
\hline 6 & 225 \\
\hline 8 & 200 \\
\hline 10 & 1000 \\
\hline 11 & 225 \\
\hline 14 & 850 \\
\hline 15 & 500 \\
\hline
\end{tabular}

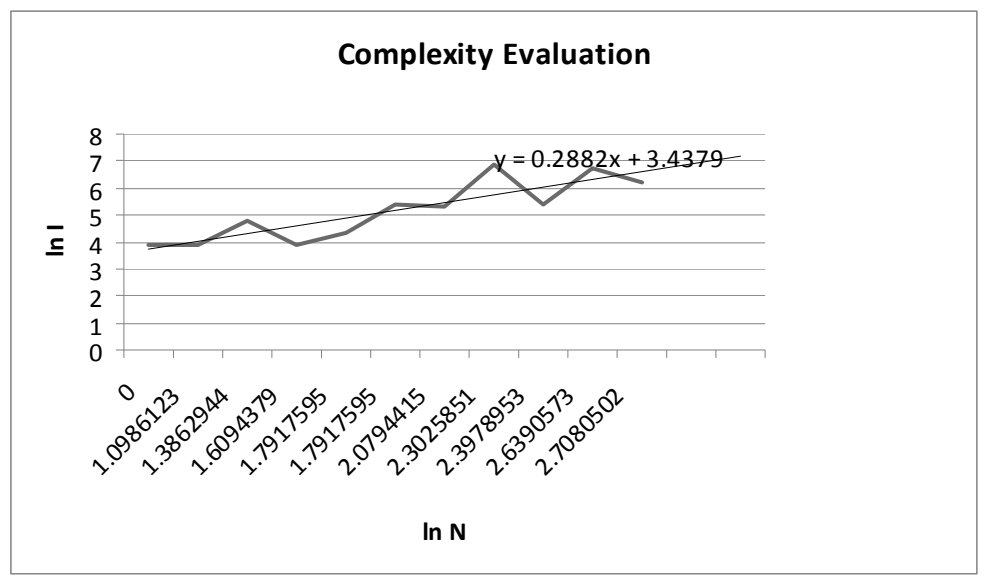

Fig. 3. Evaluation of Complexity by Linear Regression for Data in Table 1

\subsection{Evaluation of Complexity of Games Information System}

For the architecture given in Fig. 1a, for $n=2$, data throughput for a few of practical subsystems are given in Table 2 (data obtained from $[17,18,19,20]$ ).

Table 2. Components for Competition Results System in Fig. 1a

\begin{tabular}{|l|l|}
\hline Item (Data/Control Source/Sink) & Data rate \\
\hline Photo Finish System (MacFinish III Ethernet) & $100 \mathrm{Mb} / \mathrm{s}$ \\
\hline False Start Control System (ASC2) & $115 \mathrm{~kb} / \mathrm{s}$ \\
\hline $\begin{array}{c}\text { Display Control System (Nova Modular Color } \\
\text { Display) }\end{array}$ & $110 \mathrm{~kb} / \mathrm{s}$ \\
\hline $\begin{array}{c}\text { Transponder Control System (Swiss Timing using } \\
\text { GPRS technology) }\end{array}$ & $48 \mathrm{~kb} / \mathrm{s}$ \\
\hline Total: 4 & Total: $\mathbf{1 0 0 . 3 M b} / \mathbf{s}$ \\
\hline
\end{tabular}


Here we apply Algorithm 2 above and obtain, by eq. 8 ,

$$
C=2 \times \frac{\ln (100,300,000)}{\ln 4}=26.58 .
$$

In eq. 9, 100.3M has been expanded. This means that complexity is affected mostly by the high throughput subsystem and therefore special focus is needed for designing infrastructure and hardware architectures for high throughput subsystems.

\section{Observations}

Evaluation of complexity of information architectures by means of fractals gives us an approach to compare and classify software architectures. In our example evaluations, we evaluated complexity for information architectures without considering the level of the architecture in the hierarchy. Thus for the university research lab we evaluated complexity at level 1, while for the competition result system we evaluated the complexity at level 2 . We assumed that algorithms for calculating complexity are independent of the hierarchical level though this will require further study.

In our discussion we have assumed information content $(I)$ in information architectures to represent the rate of flow of data from data sources and data sinks, as well as flow of control from control sources and sinks (therefore, high data rate systems may influence computation as seen in the example in Section 3.2). However, if information is perceived as representing total data transferred or bandwidth used, then we may need to revisit equations in Section 2.

Theorem 3 equates the constant of proportionality with the initial state of architecture development. We need to investigate this further to ensure that the order in which the components are added to an architecture does not become a major factor in complexity evaluation. This is related to an assumption made in Theorem 4 where we assume the final information flow is much greater than the initial one - when this assumption fails the algorithms in Section 3 may need to be modified.

We have used one of the equations (eq. 0) possible for evaluating fractal complexity. There are other methods [11] to evaluate fractal complexity as well such as the rule complexity for generating fractals or the number of iterations needed to generate a fractal shape (indicated, to some extent, by the number $n$ in Fig. 1); we need to explore the differences in complexity evaluation for information architectures using these methods as well.

Our algorithms for assessing fractal complexity of software architectures are relatively straightforward - as new components of software architecture become known, the changes to complexity may be dynamically evaluated. This permits the software architect develop a real-time complexity view of the system being developed.

\section{Conclusion}

Complexity of software architectures is an important non-functional requirement whose quantitative values will permit system architects to better estimate maintenance effort for the final system - the more complex the architecture is, the more it typically 
costs to maintain. Information architectures for information systems capture the interconnection of components that serve as control and data sources and sinks. There is a class of information systems whose information architectures display a self-similar pattern such as, for example, campus network systems, games information systems, and the human central nervous system. These self-similar architectures are similar to fractals and in this paper we employed fractal dimension to evaluate complexity of information architecture for such systems. We developed algorithms to evaluate complexity and applied it to practical systems. In this process we found that fractalbased evaluation provides a quantitative method for dynamically (re-)computing complexity for system under development. The complexity is evaluated as numbers in this process that allows for easy comparison between software architectures. Software architects can benefit from this method by being able to compare architectures and choosing the best option for the system being developed. Also, this evaluation will help architects determine those subsystems that need special focus during infrastructure and hardware architecture designs.

There are several lines of further research in this area. It will be interesting to observe if there are differences in the complexity evaluation when applied at different hierarchical levels in a fractal information architecture. Theorem 1 gives an open upper bound for complexity- it will be useful to practitioners if complexity bands may be identified for different classes of systems. Also it will be of interest to practitioners if fractal approach could be applied to other types of software architecture such as system architecture, infrastructure architecture, or hardware architecture. However, we believe that fractal approach to complexity evaluation helps practitioners in industry and academia to quickly compute complexity for information architectures.

\section{Acknowledgement}

We thank reviewers of the earlier version of this paper for their valuable suggestions and comments. The paper has been revised to more clearly present the results of complexity analysis employing fractals.

\section{References}

1. Whitten, J.L., Bentley, L.D.: Systems Analysis and Design Methods. McGraw Hill Irwin, New York (2007)

2. Eeles, P., Cripps, P.: The Process of Software Architecting. Addison Wesley, New Jersey (2010)

3. Shaw, M., Garlan, D.: Software Architecture: Perspectives on an Emerging Discipline. Prentice Hall, New Jersey (1996)

4. International Association of Athletic Federation, Competition Rules 2010-2011, http: / / www.iaaf .org/mm/Document/Competitions/TechnicalArea/ 05/47/81/20091027115916_httppostedfile_CompRules2010_ web_260ct09_17166.pdf

5. ATOS Origin, http://www. atosorigin.com/en-us/ olympic-games/london_2012/default.htm 
6. Martini, F.H., et al.: Fundamentals of Anatomy and Physiology. Prentice Hall, New Jersey (2001)

7. McCabe, T.J.: A Complexity Measure. IEEE Transactions on Software Engineering, SE2(4), 308-320 (1976)

8. Kazman, R., Burth, M.: Assessing Architectural Complexity. In: 2nd Euromicro Conference on Software Maintenance and Reengineering, pp. 104-112. IEEE Press, Florence (1998)

9. Zhao, J.: On Assessing the Complexity of Software Architectures. In: 3rd International Workshop on Software Architecture, pp. 163-166. ACM, Orlando (1998)

10. AlSharif, M., Bond, W.P., Al-Otaiby, T.: Assessing the Complexity of Software Architecture. In: 42nd Annual Southeast Regional Conference, pp. 98-103. ACM, Huntsville (2004)

11. Mandelbrot, B.B.: The Fractal Geometry of Nature. W. H. Freeman \& Company, New York (1977)

12. Fractals and the Fractal Dimension, http://www.vanderbilt.edu/Ans/ psychology/cogsci / chaos/workshop/Fractals.html

13. Frohn, R.C.: Remote Sensing for Landscape Ecology: new metric indicators for monitoring, modeling, and Assessment of Ecosystems. CRC Press, Boca Raton (1998)

14. Fractal Dimension Index, http: / / www.trivisonno.com/fractal-dimension-index

15. O'Neill, R.V., et al.: Indices of landscape pattern. Landscape Ecology 1(3), 153-162 (1988)

16. Chung, L., Nixon, B.A., Yu, E., Mylopoulos, J.: Non-Functional Requirements in Software Engineering. Kluwer Academic Publishers, Boston (2000)

17. TimeTronics, http://www.timetronics.be/products/ macfinish.php\#MF3ETHERNET

18. Swiss Timing, http://www. swisstiming.com/ Detail.559.0.html?\&tx_stproducts_pi1[uid]=35\&tx_stproducts_ pi1 [ currentSport] =73\&tx_stproducts_pi1 [current Type] =69\&cHash $=38 a 140 d 0 e 844 d 9 c 7$ eafec $8 c c 3 c 5$ fadb9

19. Swiss Timing, http://www.swisstiming.com/uploads/ tx_stproducts/NOVA_MODULAR_COLOUR_DISPLAYS_ $3437.525 .02-S 9 . p d f$

20. Swiss Timing, http://www.swisstiming.com/Athletics.495.0.html 